\title{
Melatonin Downregulates PD-L1 Expression and Modulates Tumor Immunity in KRAS-Mutant Non-Small Cell Lung Cancer
}

\author{
Yi-Chun Chao ${ }^{1,2,+}$, Kang-Yun Lee ${ }^{3,4,5,+}$, Sheng-Ming Wu ${ }^{3,4}$, Deng-Yu Kuo ${ }^{6}$, Pei-Wei Shueng ${ }^{6,7, *(D)}$ \\ and Cheng-Wei Lin $1,2,8,9, * \mathbb{D}$
}

1 Department of Biochemistry and Molecular Cell Biology, School of Medicine, College of Medicine, Taipei Medical University, Taipei 110, Taiwan; m120109016@tmu.edu.tw

2 Graduate Institute of Medical Sciences, College of Medicine, Taipei Medical University, Taipei 110, Taiwan

3 Shuang Ho Hospital, Taipei Medical University, New Taipei City 235, Taiwan; kangyunlee68@gmail.com (K.-Y.L.); chitosan@tmu.edu.tw (S.-M.W.)

4 Division of Pulmonary Medicine, Department of Internal Medicine, School of Medicine, College of Medicine, Taipei Medical University, Taipei 110, Taiwan

5 Graduate Institute of Clinical Sciences, College of Medicine, Taipei Medical University, Taipei 110, Taiwan

6 Division of Radiation Oncology, Far Eastern Memorial Hospital, New Taipei City 220, Taiwan; dykuo@femh.org.tw

7 Faculty of Medicine, School of Medicine, National Yang Ming Chiao Tung University, Taipei 112, Taiwan

8 Cell Physiology and Molecular Image Research Center, Wan Fang Hospital, Taipei Medical University, Taipei 110, Taiwan

9 Drug Development and Value Creation Research Center, Kaohsiung Medical University, Kaohsiung 817, Taiwan

* Correspondence: shuengsir@gmail.com (P.-W.S.); cwlin@tmu.edu.tw (C.-W.L.); Tel.: +886-2-27361661 (C.-W.L.)

check for updates

Citation: Chao, Y.-C.; Lee, K.-Y.; Wu, S.-M.; Kuo, D.-Y.; Shueng, P.-W.; Lin, C.-W. Melatonin Downregulates PD-L1 Expression and Modulates Tumor Immunity in KRAS-Mutant Non-Small Cell Lung Cancer. Int. J. Mol. Sci. 2021, 22, 5649. https:// doi.org/10.3390/ijms22115649

Academic Editor: Federico Cappuzzo

Received: 5 May 2021

Accepted: 24 May 2021

Published: 26 May 2021

Publisher's Note: MDPI stays neutral with regard to jurisdictional claims in published maps and institutional affiliations.

Copyright: (c) 2021 by the authors. Licensee MDPI, Basel, Switzerland. This article is an open access article distributed under the terms and conditions of the Creative Commons Attribution (CC BY) license (https:// creativecommons.org/licenses/by/ $4.0 /)$.
+ These authors contributed equally to this work.

Abstract: Non-small cell lung cancer (NSCLC) patients harboring a KRAS mutation have unfavorable therapeutic outcomes with chemotherapies, and the mutation also renders tolerance to immunotherapies. There is an unmet need for a new strategy for overcoming immunosuppression in KRAS-mutant NSCLC. The recently discovered role of melatonin demonstrates a wide spectrum of anticancer impacts; however, the effect of melatonin on modulating tumor immunity is largely unknown. In the present study, melatonin treatment significantly reduced cell viability accompanied by inducing cell apoptosis in KRAS-mutant NSCLC cell lines including A549, H460, and LLC1 cells. Mechanistically, we found that lung cancer cells harboring the KRAS mutation exhibited a higher level of programmed death ligand 1 (PD-L1). However, treatment with melatonin substantially downregulated PD-L1 expressions in both the presence and absence of interferon (IFN)- $\gamma$ stimulation. Moreover, KRAS-mutant lung cancer cells exhibited higher Yes-associated protein (YAP) and transcriptional coactivator with PDZ-binding motif (TAZ) levels, and PD-L1 expression was positively correlated with YAP and TAZ in lung cancer cells. Treatment with melatonin effectively suppressed YAP and TAZ, which was accompanied by downregulation of YAP/TAZ downstream gene expressions. The combination of melatonin and an inhibitor of YAP/TAZ robustly decreased YAP and PD-L1 expressions. Clinical analysis using public databases revealed that PD-L1 expression was positively correlated with YAP and TAZ in patients with lung cancer, and PD-L1 overexpression suggested poor survival probability. An animal study further revealed that administration of melatonin significantly inhibited tumor growth and modulated tumor immunity in a syngeneic mouse model. Together, our data revealed a novel antitumor mechanism of melatonin in modulating the immunosuppressive tumor microenvironment by suppressing the YAP/PD-L1 axis and suggest the therapeutic potential of melatonin for treating NSCLC.

Keywords: melatonin; lung cancer; PD-L1; tumor immunity; Hippo pathway 


\section{Introduction}

Lung cancer is still the leading cause of cancer-related mortality, and non-small cell lung cancer (NSCLC) accounts for $85 \%$ of all lung cancers [1]. Oncogenic KRAS mutations are a major driver of NSCLC, accounting for over $30 \%$ of NSCLC cases in Western countries and 10\% of total lung cancer cases in Asia [2]. NSCLC patients with a KRAS mutation show a poor response to front-line chemotherapy, and they often exhibit worse prognoses. Disappointingly, unlike other oncoproteins, the mutant KRAS protein is thought to be an undruggable target due to various conformations of its mutant types [2,3]. Recently, immune checkpoint blockage through targeting programmed death-ligand 1 (PD-L1) and by its receptor programmed death-1 (PD-1), has changed the treatment paradigm for lung cancer patients, especially for those harboring a KRAS mutation [4]. KRAS mutations are often associated with high tumor mutation burdens, elevated PD-L1 expression, and an immunosuppressive tumor microenvironment [5,6]. Mutations of KRAS facilitate immune evasion by orchestrating a pro-inflammatory microenvironment [7], and evidence indicates that oncogenic RAS promotes stabilization and upregulation of PD-L1 [8]. Notably, several studies showed that patients with KRAS mutations are more sensitive to PD1/PD-L1 inhibitors [9], suggesting that the KRAS mutation may be vulnerable to immunotherapy.

Melatonin (N-acetyl-5-methoxytryptamine), an indolamine that is primarily synthesized and secreted by the pineal gland, has various biological functions such as regulation of circadian rhythm, as well as antioxidant, anti-aging, and antitumor activities [10]. There is a large amount of evidence on the benefits of melatonin in anticancer properties including its pro-apoptotic, anti-proliferative, anti-angiogenic, and anti-metastatic properties [11-13]. Melatonin also has a synergistic effect with different chemotherapeutic drugs [14-16]. In the clinical study, NSCLC patients concomitantly treated with melatonin exhibited better survival outcomes compared to those treated with chemotherapy alone [17]. Moreover, melatonin showed an anti-inflammatory response, which is known to be essential for promoting tumor development [18]. Melatonin also showed immunomodulatory activity in solid tumors [19]. Despite the antitumor activities of melatonin having been widely reported, the effect of melatonin on tumor immunity and the mechanism involved in the process remain unclear.

Yes-associated protein (YAP) and transcriptional coactivator with the PDZ-binding motif (TAZ) are transducers of the Hippo pathway, which controls organ development and whose dysregulation is involved in tumorigenesis [20,21]. YAP and TAZ are often upregulated or hyperactivated in many solid tumors including NSCLC [22,23]. Elevation of YAP/TAZ promotes stemness of NSCLC and contributes to the resistance of NSCLC to chemo- and target therapies [24,25]. On the other hand, inhibition of YAP/TAZ increases cytotoxicity of chemotherapy and sensitivity to various target therapies including EGFRTKIs and MEK inhibitors in NSCLC [26,27]. YAP/TAZ also participates in KRAS oncogenedriven pancreatic ductal carcinoma [28], and KRAS and YAP signaling pathways converge to regulate the epithelia-mesenchymal transition in lung cancer [29]. These data indicate that activation of YAP/TAZ plays a crucial role in KRAS oncogene-mediated tumorigenesis. However, no study has reported on the role of melatonin in Hippo-YAP signaling for treatment of KRAS-mutant lung cancer.

Herein, we investigated the inhibitory mechanism of melatonin against NSCLC and tumor immunity. We show for the first time that melatonin attenuates PD-L1 expression through suppressing YAP/TAZ and thereby modulating tumor immunity in KRAS-mutant NSCLC. We demonstrate that melatonin modulates an immunosuppressive tumor microenvironment, and our findings suggest a better understanding of the antitumor activity of melatonin in the surrounding tumor microenvironment.

\section{Results}

\subsection{Melatonin Reduces the Viability and Induces Apoptosis in KRAS-Mutant NSCLC Cells}

To examine the effects of melatonin on lung cancer, human NSCLC cell lines including the H460 and A549, and mouse Lewis cell carcinoma LLC1 cell lines, which harbor KRAS 
mutations, were incubated with different concentrations of melatonin for 24 and $48 \mathrm{~h}$. Results of cell viability and colony formation assays showed that melatonin dose-dependently reduced viability of lung cancer cells (Figure 1A,B). Microscopic observation showed that treatment with melatonin at $1 \mathrm{mM}$ induced cellular shrinkage, while promoting the formation of an apoptotic-like morphology under 2.5 and $5 \mathrm{mM}$ of melatonin treatment (Figure 1C). Results of flow cytometry analysis further confirmed that treatment with melatonin at 2.5 and $5 \mathrm{mM}$ concentrations increased Annexin V-positive cells (Figure 1D), and Western blot analysis showed that incubation with melatonin decreased anti-apoptotic proteins levels, including Bcl2 and Bcl-xL, but increased cleavages of Caspase 9 and Caspase 3 (Figure 1E). These data suggest that melatonin induces the death of NSCLC cells.

\subsection{Melatonin Downregulates PD-L1 Expression in KRAS-Mutant NSCLC Cells}

KRAS mutations are associated with an immunosuppressive tumor microenvironment. To evaluate the role of melatonin in modulating tumor immunity, we analyzed the level of the immune checkpoint molecule PD-L1 in lung cancer cells using the Cancer Cell Line Encyclopedia (CCLE) database. Results showed that the mRNA expression of PD-L1 (CD274) was higher in KRAS-mutant lung cancer cells than in KRAS wide-type (WT) cells (Figure 2A), and Western blot analysis showed a higher tendency of PD-L1 protein level in KRAS mutant H441 and H460 cells (Figure 2B). Importantly, treatment with melatonin dose- and time-dependently suppressed PD-L1 protein levels in H460 and A549 cells (Figure 2C). Melatonin treatment substantially decreased the PD-L1 protein level within $24 \mathrm{~h}$ (Figure 2C). Additionally, the mRNA level of PD-L1 was downregulated after incubation with melatonin for 6-12 h (Figure 2D). Moreover, pretreatment with melatonin impeded IFN $\gamma$-induced PD-L1 upregulation, as validated by Western blot and flow cytometry analyses (Figure 2E,F).

\subsection{Melatonin Downregulates the YAP/PD-L1 Axis}

To explore the inhibitory mechanism by which melatonin attenuates PD-L1 expression further, we analyzed YAP and TAZ expressions in lung cancer cells. YAP plays a crucial role in KRAS-mutation-driven tumorigenesis of pancreatic ductal adenocarcinoma [30], and we previously identified that YAP/TAZ expressions participate in the resistance of anti-EGFR therapy in KRAS-mutant NSCLC [26]. Analysis of mRNA levels from the CCLE database showed that the expressions of YAP (YAP1) and TAZ (WWTR1) were upregulated in KRAS-mutant lung cancer cells, compared to KRAS wild-type tumor cells (Figure 3A). Moreover, expression of PD-L1 positively correlated with YAP/TAZ in lung cancer cells (Figure 3B). We further analyzed protein levels of YAP/TAZ in lung cancer cell lines, and the results showed that YAP and TAZ were not exclusively expressed in KRAS-mutant lung cancer cells (Figure $3 C$ ), suggesting that other molecules apart from KRAS may regulate protein levels of YAP/TAZ. Nevertheless, we found that treatment with melatonin at 1-2.5 mM concentrations for $24 \mathrm{~h}$ induced phosphorylation of YAP and was accompanied with a reduction in total YAP protein level by $5 \mathrm{mM}$ melatonin treatment in both H460 and A549 cells (Figure 3D). The phosphorylated YAP induces cytoplasmic retention and subsequently facilitates proteasomal degradation. In addition, we also found that treatment with melatonin substantially inhibited TAZ protein levels in H460 and A549 cells (Figure 3D). Results of real-time PCR assays also showed that melatonin downregulated mRNA levels of YAP/TAZ downstream targets including CTGF and Cyr61 (Figure 3E). Additionally, we validated that treatment with the inhibitor of YAP/TAZ by verteporfin (VP) caused robust decreases in YAP and PD-L1 protein levels, and co-treatment with VP and melatonin enhanced the inhibitory effect, as measured by Western blot and flow cytometry analyses (Figure 3F,G). Furthermore, examination of a TCGA lung adenocarcinoma cohort validated that PD-L1 (CD274) was positively correlated with YAP (YAP1), TAZ (WWTR1), and the YAP/TAZ signature (Figure 3H), and NSCLC patients with high PD-L1 expression conferred poor survival probabilities (Figure 3I). These data suggest that elevation of YAP/TAZ in KRAS-mutant NSCLC plays a role in PD-L1 
regulation, and melatonin effectively suppressed the YAP/PD-L1 axis in KRAS-mutant NSCLC cells.
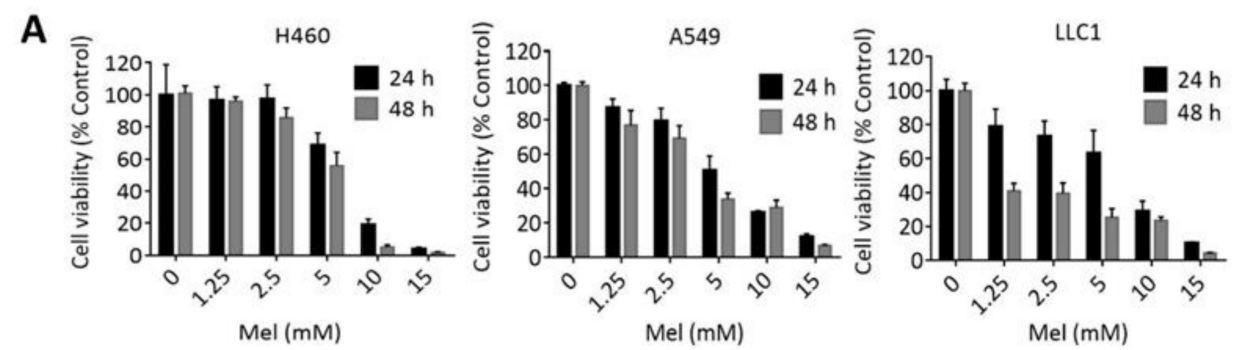

\section{B}
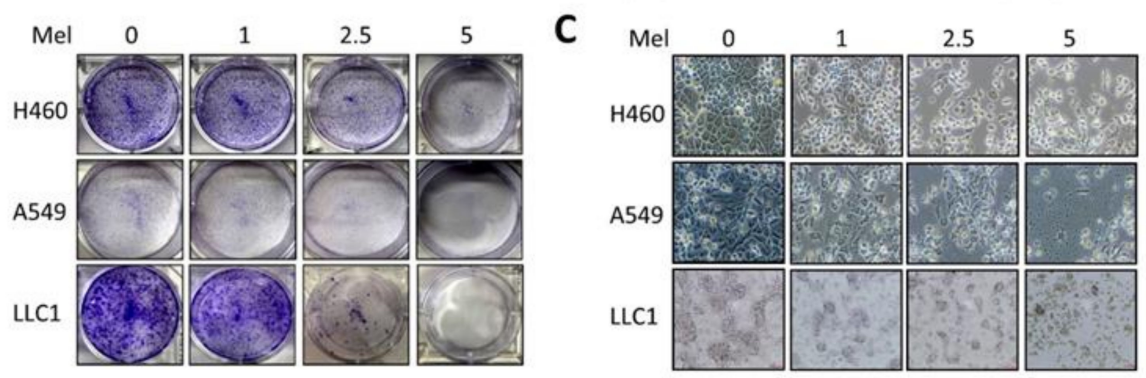

D

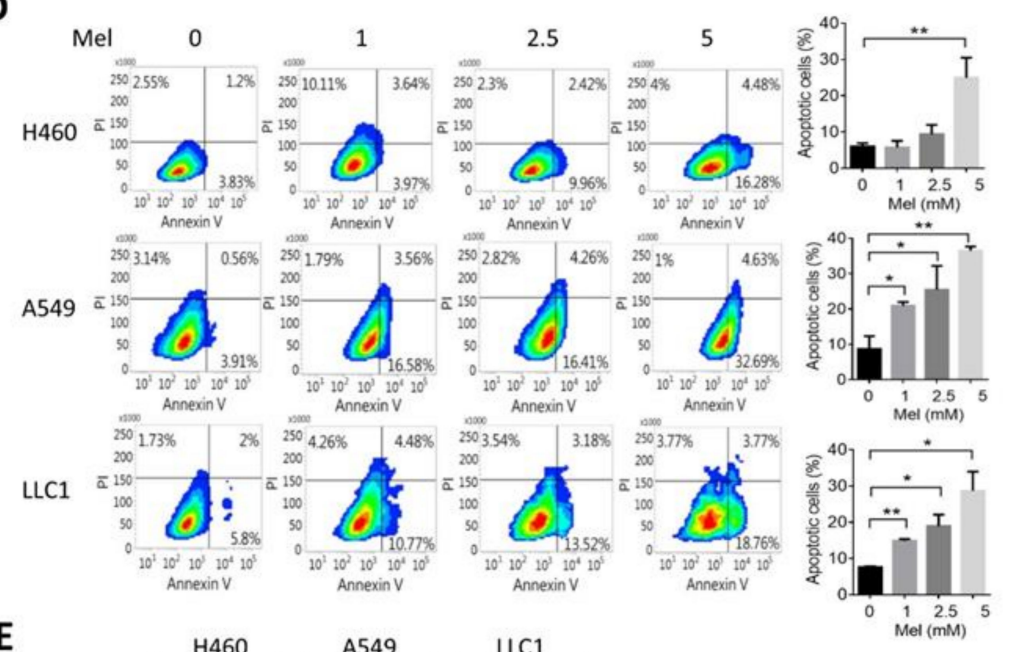

E

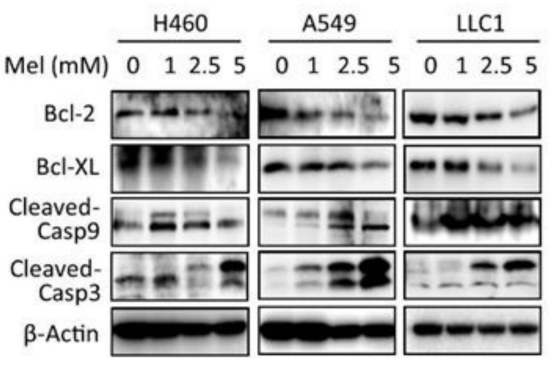

Figure 1. Effect of melatonin on the viability of NSCLC. (A) H460, A549, and LLC1 cells were treated with various concentrations of melatonin (Mel: 0, 1.25, 2.5, 5, 10, $15 \mathrm{mM}$ ) for 24 and $48 \mathrm{~h}$, and cell viability was measured with a CCK8 assay. Data are expressed as a percentage of the control. Values are expressed as the mean \pm standard deviation. (B) Colony formation of H460, A549, and LLC1 cells in the presence of melatonin (Mel: 0, 1, 2.5, $5 \mathrm{mM}$ ) for 7 days. (C) Microscopic observation of cell morphologies of H460, A549, and LLC1 cells in the presence of melatonin (Mel: 0, 1, 2.5, $5 \mathrm{mM}$ ) for $24 \mathrm{~h}$. (D) Flow cytometry analysis of Annexin V/PI staining in response to melatonin (Mel: 0, 1, 2.5, $5 \mathrm{mM}$ ) for $24 \mathrm{~h}$. Quantifications of the total apoptotic cell population (Annexin V + /PI- and Annexin $\mathrm{V}+/ \mathrm{PI}+$ ) were obtained from three independent experiments (right panel). Data are presented as the mean \pm standard deviation (SD). ${ }^{*} p<0.05,{ }^{* *} p<0.01$, as determined by an unpaired $t$-test. (E) Western blot analysis of apoptosis-related proteins in H460, A549, and LLC1 cells in response to melatonin (Mel: $0,1,2.5,5 \mathrm{mM}$ ) for 24 or $48 \mathrm{~h}$. 
A

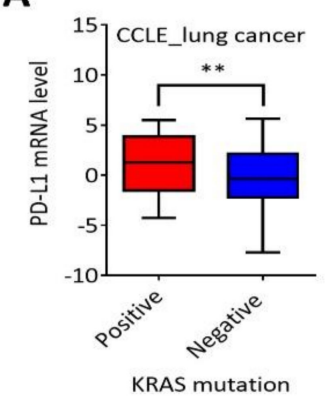

B

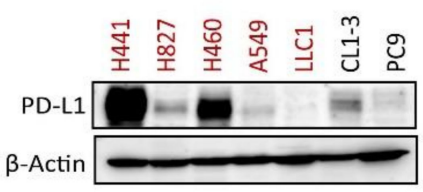

C

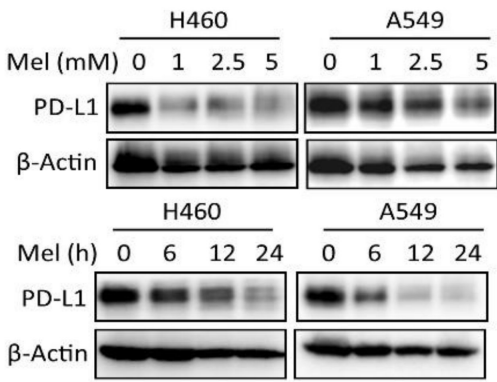

D
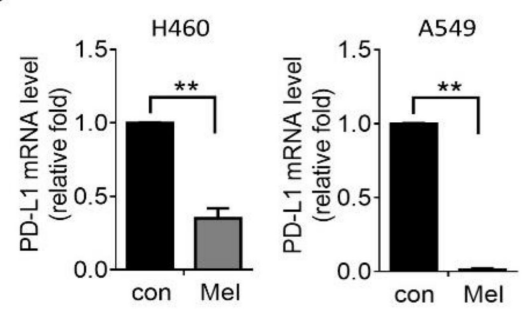

$\mathbf{E}$

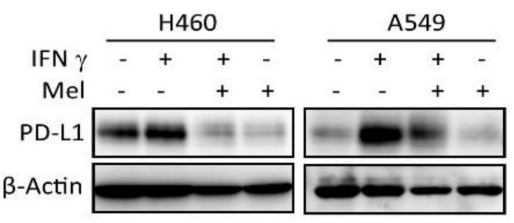

$\mathbf{F}$
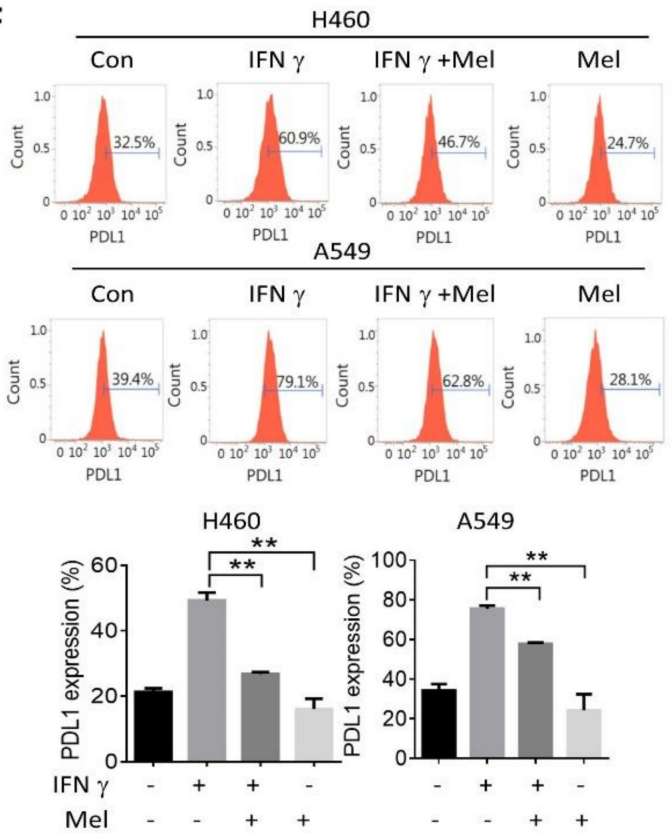

Figure 2. Melatonin downregulates PD-L1 in NSCLC. (A) Box plot with Tukey whisker of PD-L1 mRNA level in KRAS mutant and wild-type lung cancer cells. ${ }^{* *} p<0.01$ as determined by an unpaired $t$-test. (B) Western blot analysis of PD-L1 protein level in NSCLC cell lines. KRAS-mutant cell lines were labeled in red color. (C) $\mathrm{H} 460$ and A549 cells were treated with various concentrations of melatonin (Mel: 0, 1, 2.5, $5 \mathrm{mM}$ ) for $24 \mathrm{~h}$ (upper panel), or treated with $2.5 \mathrm{mM}$ melatonin at different time intervals (lower panel), and protein lysates were subjected to Western blot analysis. (D) Real-time PCR analysis of PD-L1 mRNA expression in response to melatonin (Mel: $2.5 \mathrm{mM}$ ) for 6 and $12 \mathrm{~h}$ in $\mathrm{A} 549$ and $\mathrm{H} 460$ cells, respectively. Values are expressed as the mean \pm standard deviation. ** $p<0.01$ was regarded as indicating a significant difference. (E and F) Serum-starved H460 and A549 cells were pretreated with melatonin $(2.5 \mathrm{mM})$ for $60 \mathrm{~min}$ followed by stimulation with IFN $\gamma(50 \mathrm{ng} / \mathrm{mL})$ for a further $24 \mathrm{~h}$. (E) Protein level of PD-L1 in total cell lysate was measured by Western blot, and (F) histogram plot of PD-L1 expression was performed by flow cytometry analyses. Quantifications of PD-L1 positive percentages from three-independent experiments are shown (lower panel). ${ }^{* *} p<0.01$, as determined by an unpaired $t$-test. 
A

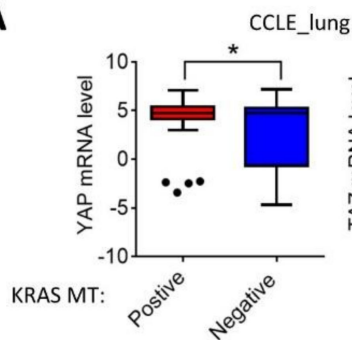

B

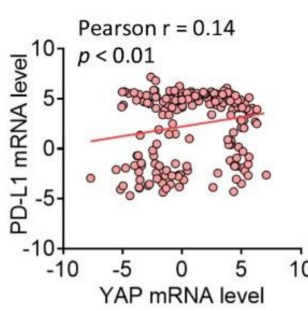

$\mathbf{E}$

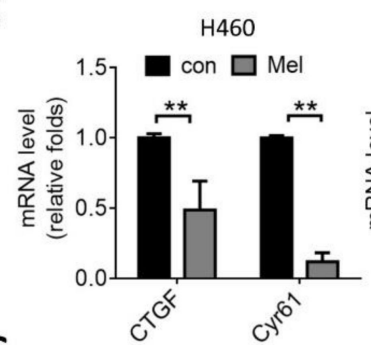

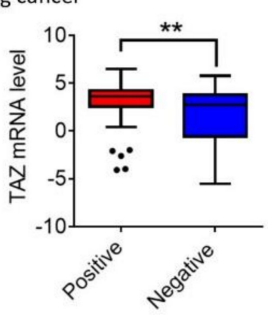

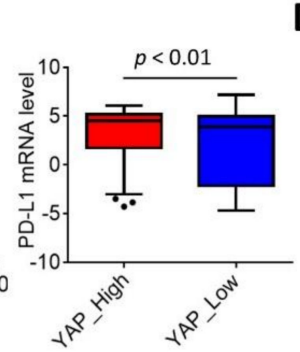

C

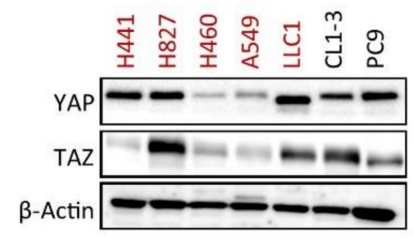

D

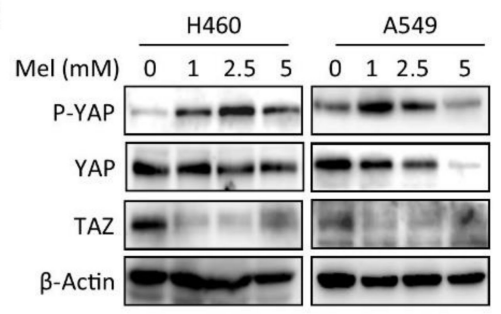

$\mathbf{F}$

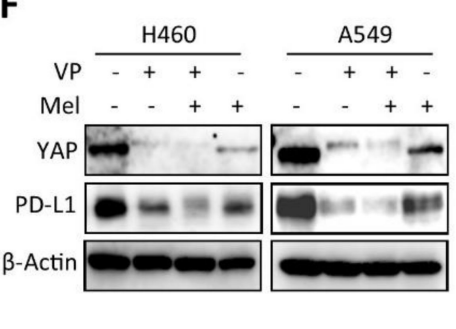

G

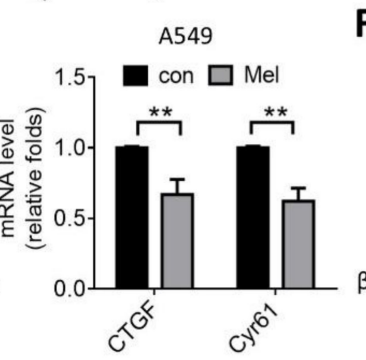

A549
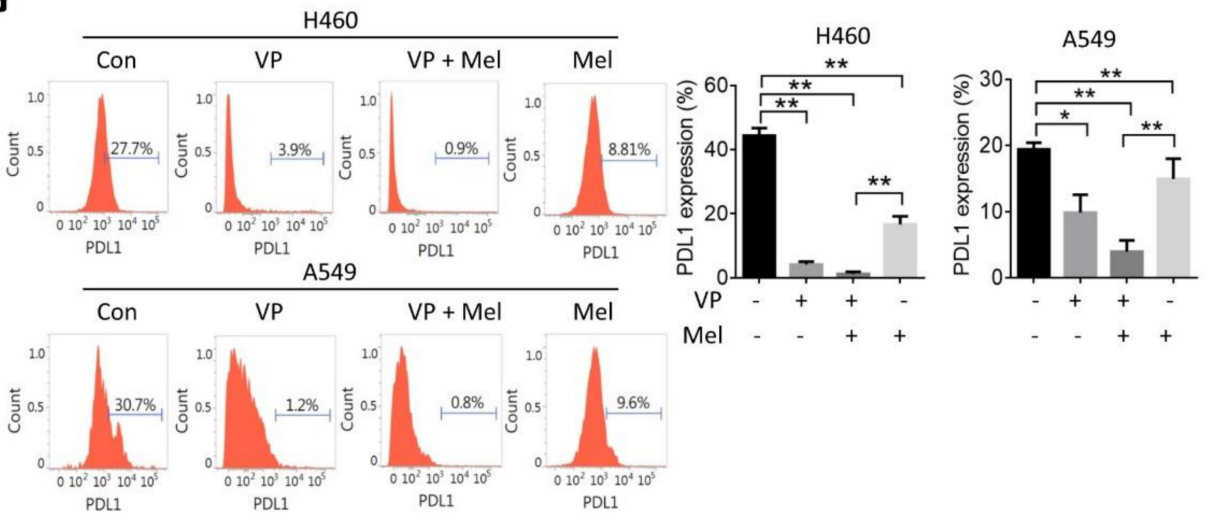

H
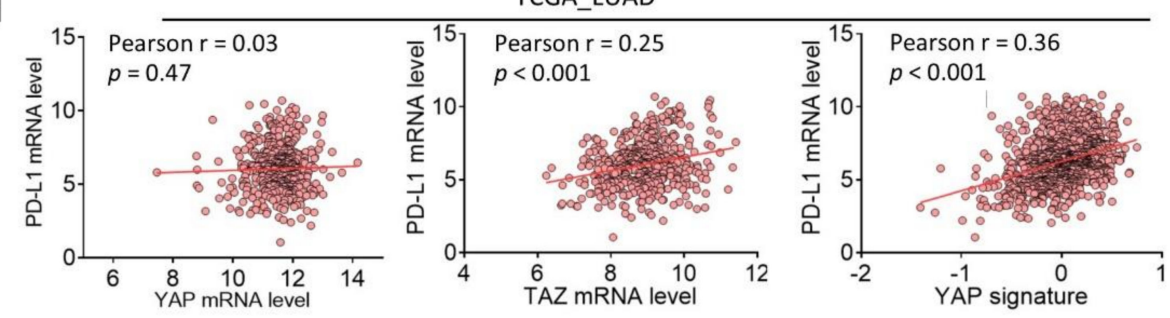

I

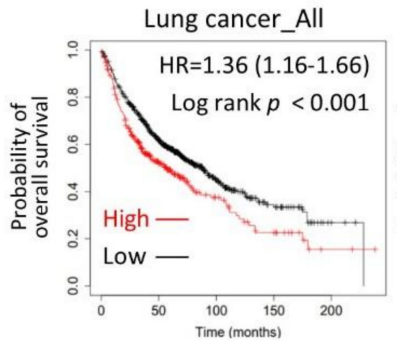

LUAD
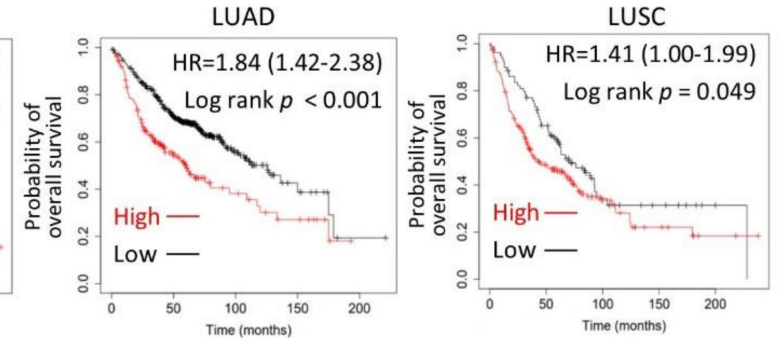

Figure 3. Melatonin inhibits the YAP/PD-L1 axis. (A) Box plots with Tukey whisker of YAP (YAP1) and TAZ (WWTR1) mRNA levels in KRAS-mutant and wild-type lung cancer cell lines. ${ }^{*} p<0.05$ and ** $p<0.01$ as determined by an unpaired $t$-test. (B) Scatterplot of PD-L1 (CD274) and YAP (YAP1) in 
lung cancer cell lines (left panel). Correlation coefficient was performed by Pearson's test. Box blot of PD-L1 (CD274) levels in lung cancer cell lines stratified by YAP (YAP1) expression levels (right panel). (C) Western blot analysis of YAP and TAZ protein levels in NSCLC cell lines. KRAS-mutant cell lines were labeled in red color. (D) $\mathrm{H} 460$ and A549 cells were treated with various concentrations of melatonin (Mel: 0, 1, 2.5, $5 \mathrm{mM}$ ) for $24 \mathrm{~h}$, and protein lysates were subjected to Western blot analysis. (E) RT-qPCR analysis of the expressions of CTGF and Cyr61 in melatonin-treated H460 and A549 cells. ${ }^{* *} p<0.01$ as determined by an unpaired $t$-test. (F) Western blot analysis of YAP and PD-L1 protein levels in H460 and A549 cells treated with verteporfin (VP: $1 \mu \mathrm{M}$ ) and melatonin (Mel: $2.5 \mathrm{mM}$ ). (G) Flow cytometry analysis of PD-L1 expression in H460 and A549 treated with verteprofin (VP: $1 \mu \mathrm{M}$ ) and melatonin (Mel: $2.5 \mathrm{mM}$ ). Quantifications of PD-L1 positive percentage from three-independent experiments are shown. ${ }^{*} p<0.05$ and ${ }^{* *} p<0.01$, as determined by a one-way ANOVA. (H) Scatterplot of PD-L1 (CD274) and YAP (YAP1), TAZ (WWTR1), and YAP/TAZ signature in TCGA_LUAD cohort. Correlation coefficients were determined by Pearson's test. (I) KaplanMeier curve analysis of the overall survival probability of lung cancer patients stratified by PD-L1 (CD274) expression level. LUAD: lung adenocarcinoma; LUSC: lung squamous cell carcinoma. HR: hazard ratio.

\subsection{Melatonin Suppresses Tumor Growth and Exerts Antitumor Immunity}

We next evaluated the effect of melatonin on tumor immunity using a syngeneic mouse model. B57CL/ 6 mice were subcutaneously inoculated with LLC1 tumor cells and administered melatonin (30 mg/kg, three times per week) for a total of four weeks. Results showed that mice receiving melatonin exhibited effective inhibition of tumor growth (Figure 4A,B), whereas the mice body weights did not significantly change during melatonin administration (Figure 4C). Notably, analyses of tumor-infiltrating lymphocytes indicated that treatment with melatonin significantly increased the number of CD3 + CD4+ and CD3 + CD8+ T cells, but reduced infiltration of Ly6G + F4/80- myeloid-derived suppressor cells (MDSC). Melatonin treatment slightly but insignificantly reduced F4/80+ tumor-associated macrophages (TAM) (Figure 4D and Figure S1). These data indicate that melatonin may suppress tumor growth and ameliorate the immunosuppressive tumor microenvironment.

A

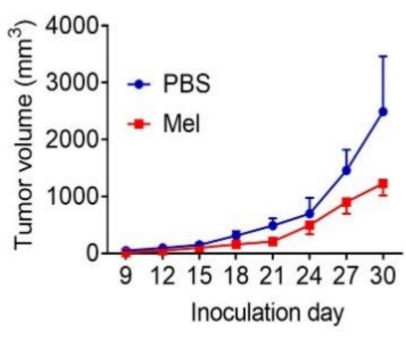

B

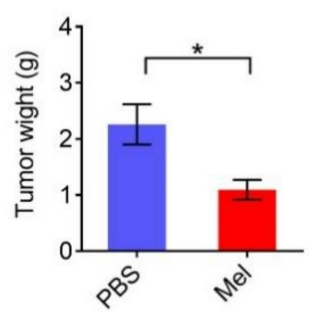

C

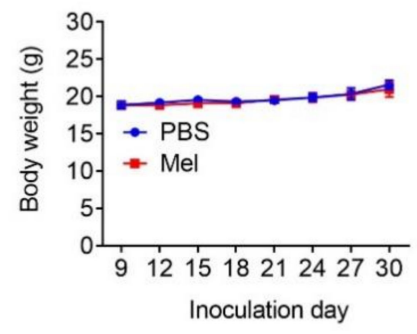

D
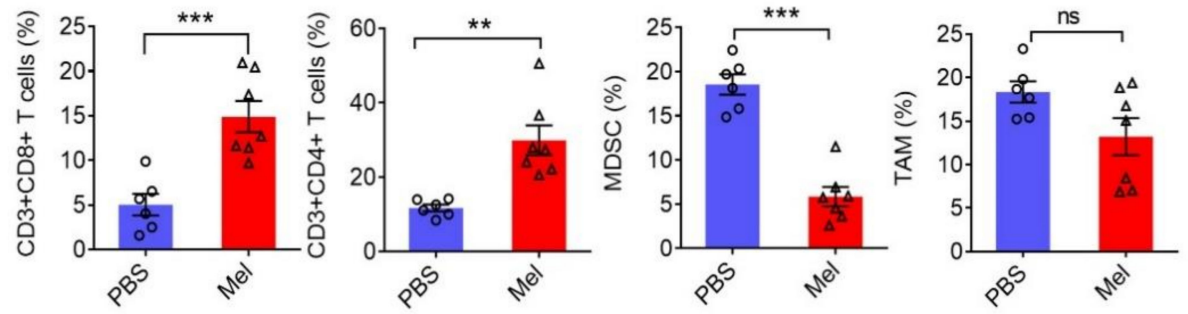

Figure 4. Effects of melatonin on tumor growth and tumor immunity. (A) Growth curve of LLC1 tumor-bearing mice administrated PBS $(n=6)$ or melatonin $(30 \mathrm{mg} / \mathrm{kg}$, three times per week, $n=7$ ) for 4 weeks. Tumor volumes were measured every other day. (B) Tumor weights were measured at the end of the experiment. (C) Mouse body weight changes under different treatments. (D) Quantification of tumor-infiltrating lymphocytes in PBS- and melatonin-treated tumor tissues, as measured by flow cytometry analysis. Values are expressed as the mean \pm standard deviation. ${ }^{*} p<0.05,{ }^{* *} p<0.01,{ }^{* * *} p<0.001$ indicating a significant difference. 


\section{Discussion}

Immunotherapies targeting PD-L1 or its receptor PD1, have shown benefits in patients with advanced lung cancer. Evidence indicates that oncogenic RAS promotes stabilization of PD-L1, and KRAS mutations upregulate PD-L1 and mediate immune escape in NSCLC [8,31]. Although KRAS mutations facilitate immune evasion by promoting a proinflammatory microenvironment, several clinical studies also reported that patients with KRAS mutations were more sensitive to PD1/PD-L1 inhibitors, suggesting vulnerability to anti-PD1/PD-L1 therapy by patients with KRAS mutations. In our study, the PD-L1 mRNA expression was associated with mutant KRAS by the CCLE database; however, protein levels of PD-L1 were not exclusively expressed in mutant KRAS cells. Several studies and meta-analyses have confirmed the correlation between PD-L1 expression and KRAS status in NSCLC [32-35], but conflicting studies reported that expression of PD-L1 was varied according to the pattern of downstream signaling [36,37]. Moreover, other oncogenic drivers such as BRAF, PIK3CA, and MET showed more frequent PD-L1 expression [38]. Therefore, it is warranted to clarify the regulation of PD-L1 expression in NSCLC.

Possible crosstalk between melatonin and the Hippo-YAP signaling pathway involves the G protein-coupled receptor (GPCR), which is mainly associated with the melatonin receptor and is also involved in the regulation of the upstream regulators of YAP/TAZ, such as LATS1/2 and F-actin [39]. Previous studies reported that melatonin protects against lung fibrosis by inhibiting the Hippo-YAP pathway [40]. The effect of melatonin on the YAP/TAZ pathway in lung cancer remains unclear. In the present study, we identified that YAP/TAZ expressions were upregulated in KRAS-mutant lung cancer cells. Moreover, we demonstrated that melatonin effectively suppressed YAP/TAZ in KRAS-mutant A549 and H460 cells, and melatonin downregulated the YAP/TAZ downstream genes' expression. Additionally, we found that melatonin induced phosphorylation of YAP. Phosphorylation of YAP and TAZ by LATS1/2 results in their cytoplasmic retention and facilitates proteasomal degradation. Because YAP/TAZ also play crucial role in promoting tumor growth, the inhibition of YAP/TAZ results in inducing cell death $[25,26,41]$. Therefore, downregulation of YAP/TAZ may contribute to melatonin-induced growth inhibition. Nevertheless, it is still unclear whether melatonin affects upstream molecules of the Hippo cascade which is worth exploring.

Activation of YAP/TAZ has been reported to regulate PD-L1 transcription and immune escape [42], while suppression of the Hippo-YAP signaling attenuates PD-L1 expression and shows promise in ameliorating immune evasion [43]. It has been reported that melatonin can stimulate nature killer (NK) cells and modulate regulatory $\mathrm{T}$ (Treg) cells, and melatonin promotes Th1 in tumor-bearing mice [44-46]. A previous study reported that incubation of macrophages with exosome from melatonin-treated hepatocarcinoma cells downregulated PD-L1 and proinflammatory cytokines' expressions [47]. The combined use of melatonin and an indoleamine 2,3-dioxygenase-1 inhibitor improved the efficacy of immunotherapy (gDE7) targeting human papillomavirus (HPV)-associated tumors [48]. Moreover, melatonin suppresses pro-inflammatory responses; thus, melatonin also plays a crucial role in promoting the development of an immunosuppressive tumor microenvironment [49]. However, the effect of melatonin against tumor PD-L1 expression and the underlying mechanism remain unclear. In the present study, we demonstrated that melatonin suppressed YAP/TAZ which was accompanied by inhibition of PD-L1. Concomitant treatment with melatonin and a YAP/TAZ inhibitor synergistically suppressed PD-L1 expression in NSCLC cells. The in vivo study revealed that melatonin suppressed the expressions of YAP and PD-L1 in tumor tissues, and administration of melatonin decreased infiltrations of TAM and MDSC while increasing CD8+ and CD4+ T cells. Because melatonin shows a broad synergistic benefit with chemotherapies, the therapeutic efficacy of melatonin as an adjuvant, in combination with immunotherapies such as anti-PD1 or anti-PD-L1, envisions a promising approach for lung cancer treatment, and future research is warranted to investigate. 


\section{Materials and Methods}

\subsection{Cell Culture}

The human A549, H460, PC9, H827, H441, and mouse Lewis cell carcinoma LLC1 NSCLC cell lines were purchased from the Bioresource Collection Research Center (BCRC, Hsinchu, Taiwan) or American Type Culture Collection (ATCC, Manassas, VA, USA). Genetic identities of the cell lines were authenticated through short tandem repeat profiling (GenePrint 10 System). Cells were maintained in the Roswell Park Memorial Institute (RPMI) 1640 medium supplemented with 7\% fetal bovine serum (FBS), 1\% penicillinstreptomycin, and 1\% Glutagro (Corning, Corning, NY, USA). All cells were cultured at $37^{\circ} \mathrm{C}$ and in a humidified atmosphere of $5 \% \mathrm{CO}_{2}$.

\subsection{Cell Viability Assay}

NSCLC cells $\left(6 \times 10^{3}\right.$ cells/well) were seeded into 96-well plates and treated with different concentrations of melatonin (Selleck) for 24 and $48 \mathrm{~h}$. After incubation, cells were fed growth medium containing the Cell Counting Kit-8 (CCK-8) reagent (Dojindo Molecular Technologies) for $60 \mathrm{~min}$, and the formazan dye was measured with a spectrophotometer at $450 \mathrm{~nm}$ absorbance. Cell viability is expressed as the percentage of untreated control cells.

\subsection{Flow Cytometry}

To detect cell apoptosis, NSCLC cells $\left(5 \times 10^{5}\right.$ cells/well) were seeded into 6-well plates and treated with melatonin for $24 \mathrm{~h}$. Cells were trypsinized followed by incubation with an Annexin V-FITC/propidium iodide (PI) Apoptosis detection kit (Elabscience) according to the manufacturer's instruction. To measure PD-L1 protein levels, cells were treated with melatonin $(2.5 \mathrm{mM})$ in the presence or absence of IFN $\gamma(50 \mathrm{ng} / \mathrm{mL})$ for $24 \mathrm{~h}$. Cells were then trypsinized followed by incubation with a PE-conjugated anti-PD-L1 antibody (Abcam) and analyzed with a BD FACSVia flow cytometer (BD Biosciences).

\subsection{Western Blotting}

Cells were lysed in an ice-cold radioimmunoprecipitation assay (RIPA) buffer supplemented with a protease and phosphatase inhibitor cocktail (Roche, Mannheim, German). Equal amounts of protein were separated by sodium dodecylsulfate (SDS)-polyacrylamide gel electrophoresis (PAGE) and transferred to polyvinylidene difluoride membranes (Millipore, Bedford, MA, USA). Membranes were blocked with $1 \%$ bovine serum albumin/TBST blocking buffer at room temperature for $30 \mathrm{~min}$ and then incubated overnight at $4{ }^{\circ} \mathrm{C}$ with specific primary antibodies. Primary antibodies for YAP (\#12395), YAP/TAZ (\#8410), phospho-YAP(S127) (\#13008), Caspase 9 (\#9504), and PD-L1 (\#13684) were purchased from Cell Signaling Technology (Danvers, MA, USA). Antibodies for $\beta$-actin (GTX109639), Bcl-2 (GTX100064), Bcl-xL (GTX105661), Caspase 3 (GTX110543), and Caspase 9 (GTX112888) were obtained from GeneTex (San Antonio, TX, USA). Membranes were washed three times with the TBST wash buffer followed by incubation with a horseradish peroxidaseconjugated secondary antibody (Jackson ImmunoResearch, West Grove, PA, USA) at room temperature for $1 \mathrm{~h}$. Bands were detected with an enhanced chemiluminescence (ECL) system (Millipore, Bedford, MA, USA). Western blotting was performed at least three times, and representative experiments are shown.

\subsection{RNA Isolation and Real-Time Quantitative Polymerase Chain Reaction}

Total RNA was extracted with a GENzolTM TriRNA Pure kit (Geneaid, Taipei, Taiwan). cDNA was synthesized with M-MLV reverse transcriptase (Promega) and amplified with the GoTaq qPCR Master Mix (Promega) in a StepOne Plus Real-Time PCR system (Applied Biosystems, Darmstadt, Germany) with specific primers as follows: Cyr61, 5' GAGTGGGTC TGTGAC GAGGAT-3'(sense), and 5'-GGTTGTATAGGATGCGAGGCT$3^{\prime}$ (antisense); CTGF, $5^{\prime}$-CTGTGCAGCATGGACGTT-3' (sense), and 5'-GGCAGC TTGACCCTCCTC-3' (antisense); PD-L1, 5'-GTGGCATCCAAGATACAAACTCAA-3' (sense), and 
$5^{\prime}$-TCCTTCCTCTTGTCACGCTCA-3' (antisense). Results were calculated using the $\Delta \Delta C T$ equation and are expressed as multiples of change relative to a control sample [50].

\subsection{Animal Study}

All animals were cared for in a specific pathogen-free room and treated following the animal care protocol approved by an animal committee. Mouse LLC1 $\left(1 \times 10^{6}\right)$ cells were subcutaneously injected into 8-week-old -C57BL/ 6 mice. After one week of inoculation, the mice were intraperitoneally administered melatonin $(30 \mathrm{mg} / \mathrm{kg}$, three times per week) for 4 weeks. The tumor volume and mice body weights were monitored every 2 days during the experiments. Tumor volume was calculated using the following equation: length $x$ (width) $2 \times 0.5$. To analyze tumor-infiltrating lymphocytes (TILs), freshly isolated tumor tissue was cut into small pieces and disassociated by the gentleMACS tumor dissociation kit (Miltenyi Biotec). The suspension was further treated with a RBC lysis buffer to remove red blood cells. Approximate $1 \times 10^{6}$ isolated cells were incubated with fluorophoreconjugated antibodies including CD45-APC, CD11b-PE, CD3-FITC, CD8-APC-Vio770, Ly6G-FITC, CD4-PE-Vio770, and F4/80-APC (Miltenyi Biotec) and analyzed by the BD FACSVia flow cytometer (BD Biosciences) [43].

\subsection{Statistical Analyses}

Gene expression patterns of PD-L1 (CD274), YAP (YAP1), and TAZ (WWTR1) were downloaded from the University of California, Santa Cruz (UCSC) Xena browser (https: / / xenabrowser.net/ accessed on 5 May 2021) and Cancer Cell Line Encyclopedia (CCLE) (https://portals.broadinstitute.org/ccle accessed on 5 May 2021). The unit of mRNA in CCLE is $\log 2$ normalized RNA expression, and the unit of gene expression in the TCGA dataset is (norm_count + 1). The YAP signature was defined by using the CORDENONSI_YAP_CONSERVED signature from a gene set enrichment analysis (GSEA). Survival prognoses of PD-L1 (CD274) in lung cancer patients were evaluated using the KM plotter (https://kmplot.com accessed on 5 May 2021). Data are presented as the mean \pm standard deviation (SD) of three independent experiments. Statistical significance was determined by an unpaired, two-tailed Student's $t$-test unless stated otherwise. ${ }^{*} p<0.05$ and ${ }^{* *} p<0.01$ indicate statistical significance. Correlation coefficients were analyzed by Pearson's test. All statistical analyses were carried out using GraphPad Prism 6.0 software.

\section{Conclusions}

Taken together, our findings provide a novel understanding of the antitumor activity of melatonin by suppressing the YAP/PD-L1 axis and enhancing antitumor immunity. Because melatonin shows broad synergistic benefits with chemotherapies, the therapeutic potentiality of melatonin as an adjuvant in combination with immunotherapy envisions a promising approach for cancer treatment.

Supplementary Materials: The following are available online at https:/ /www.mdpi.com/article/10 .3390/ijms22115649/s1, Figure S1: Gating Strategy for tumor-infiltrating lymphocytes.

Author Contributions: Study design and conduct: Y.-C.C., K.-Y.L., S.-M.W., and P.-W.S. Data collection: Y.-C.C., K.-Y.L., S.-M.W., D.-Y.K., and P.-W.S. Data analysis and interpretation: Y.-C.C., K.-Y.L., and S.-M.W. Drafting of the manuscript: C.-W.L. Revising manuscript content and approving the final version of manuscript: C.-W.L. All authors have read and agreed to the published version of the manuscript.

Funding: This study was supported by grants from the Ministry of Science and Technology, Taiwan (MOST106-2320-B-038-040 and MOST107-2320-B-038-052-MY3 to CWL), Taipei Medical University and Shuang Ho Hospital (109TMU-SHH-17 to KYL), and Far Eastern Memorial Hospital (FEHM2021-C-06 to PWS). 
Institutional Review Board Statement: All animal studies were performed according to guidelines and with approval of the Animal Care and Use Committee of Taipei Medical University. (protocol \#LAC-2018-0255).

Informed Consent Statement: Not applicable.

Data Availability Statement: Not applicable.

Conflicts of Interest: The authors declare no conflict of interest.

\section{References}

1. Ferlay, J.; Colombet, M.; Soerjomataram, I.; Parkin, D.M.; Pineros, M.; Znaor, A.; Bray, F. Cancer statistics for the year 2020: An overview. Int. J. Cancer 2021. [CrossRef] [PubMed]

2. Ferrer, I.; Zugazagoitia, J.; Herbertz, S.; John, W.; Paz-Ares, L.; Schmid-Bindert, G. KRAS-Mutant non-small cell lung cancer: From biology to therapy. Lung Cancer 2018, 124, 53-64. [CrossRef] [PubMed]

3. Uras, I.Z.; Moll, H.P.; Casanova, E. Targeting KRAS Mutant Non-Small-Cell Lung Cancer: Past, Present and Future. Int. J. Mol. Sci. 2020, 21, 4325. [CrossRef] [PubMed]

4. Hamarsheh, S.; Gross, O.; Brummer, T.; Zeiser, R. Immune modulatory effects of oncogenic KRAS in cancer. Nat. Commun. 2020, 11, 5439. [CrossRef]

5. Lee, C.K.; Man, J.; Lord, S.; Cooper, W.; Links, M.; Gebski, V.; Herbst, R.S.; Gralla, R.J.; Mok, T.; Yang, J.C. Clinical and Molecular Characteristics Associated With Survival Among Patients Treated With Checkpoint Inhibitors for Advanced Non-Small Cell Lung Carcinoma: A Systematic Review and Meta-analysis. JAMA Oncol. 2018, 4, 210-216. [CrossRef]

6. Schoenfeld, A.J.; Rizvi, H.; Bandlamudi, C.; Sauter, J.L.; Travis, W.D.; Rekhtman, N.; Plodkowski, A.J.; Perez-Johnston, R.; Sawan, P.; Beras, A.; et al. Clinical and molecular correlates of PD-L1 expression in patients with lung adenocarcinomas. Ann. Oncol. 2020, 31, 599-608. [CrossRef]

7. Kitajima, S.; Thummalapalli, R.; Barbie, D.A. Inflammation as a driver and vulnerability of KRAS mediated oncogenesis. Semin Cell Dev. Biol. 2016, 58, 127-135. [CrossRef]

8. Coelho, M.A.; de Carne Trecesson, S.; Rana, S.; Zecchin, D.; Moore, C.; Molina-Arcas, M.; East, P.; Spencer-Dene, B.; Nye, E.; Barnouin, K.; et al. Oncogenic RAS Signaling Promotes Tumor Immunoresistance by Stabilizing PD-L1 mRNA. Immunity 2017, 47, 1083-1099.e1086. [CrossRef]

9. Liu, C.; Zheng, S.; Jin, R.; Wang, X.; Wang, F.; Zang, R.; Xu, H.; Lu, Z.; Huang, J.; Lei, Y.; et al. The superior efficacy of anti-PD-1/PD-L1 immunotherapy in KRAS-mutant non-small cell lung cancer that correlates with an inflammatory phenotype and increased immunogenicity. Cancer Lett. 2020, 470, 95-105. [CrossRef]

10. Pourhanifeh, M.H.; Sharifi, M.; Reiter, R.J.; Davoodabadi, A.; Asemi, Z. Melatonin and non-small cell lung cancer: New insights into signaling pathways. Cancer Cell Int. 2019, 19, 131. [CrossRef]

11. Wang, S.W.; Tai, H.C.; Tang, C.H.; Lin, L.W.; Lin, T.H.; Chang, A.C.; Chen, P.C.; Chen, Y.H.; Wang, P.C.; Lai, Y.W.; et al. Melatonin impedes prostate cancer metastasis by suppressing MMP-13 expression. J. Cell Physiol. 2021, 236, 3979-3990. [CrossRef] [PubMed]

12. Gurunathan, S.; Qasim, M.; Kang, M.H.; Kim, J.H. Role and Therapeutic Potential of Melatonin in Various Type of Cancers. Onco Targets Ther. 2021, 14, 2019-2052. [CrossRef]

13. Bhattacharya, S.; Patel, K.K.; Dehari, D.; Agrawal, A.K.; Singh, S. Melatonin and its ubiquitous anticancer effects. Mol. Cell Biochem. 2019, 462, 133-155. [CrossRef] [PubMed]

14. Liao, Y.; Gao, Y.; Chang, A.; Li, Z.; Wang, H.; Cao, J.; Gu, W.; Tang, R. Melatonin synergizes BRAF-targeting agent dabrafenib for the treatment of anaplastic thyroid cancer by inhibiting AKT/hTERT signalling. J. Cell Mol. Med. 2020, 24, 12119-12130. [CrossRef] [PubMed]

15. Li, M.; Wu, C.; Muhammad, J.S.; Yan, D.; Tsuneyama, K.; Hatta, H.; Cui, Z.G.; Inadera, H. Melatonin sensitises shikonin-induced cancer cell death mediated by oxidative stress via inhibition of the SIRT3/SOD2-AKT pathway. Redox Biol. 2020, $36,101632$. [CrossRef]

16. Zhou, B.; Lu, Q.; Liu, J.; Fan, L.; Wang, Y.; Wei, W.; Wang, H.; Sun, G. Melatonin Increases the Sensitivity of Hepatocellular Carcinoma to Sorafenib through the PERK-ATF4-Beclin1 Pathway. Int. J. Biol. Sci. 2019, 15, 1905-1920. [CrossRef]

17. Lissoni, P.; Chilelli, M.; Villa, S.; Cerizza, L.; Tancini, G. Five years survival in metastatic non-small cell lung cancer patients treated with chemotherapy alone or chemotherapy and melatonin: A randomized trial. J. Pineal Res. 2003, 35, 12-15. [CrossRef]

18. Ma, S.; Zhu, L.; Fan, X.; Luo, T.; Liu, D.; Liang, Z.; Hu, X.; Shi, T.; Tan, W.; Wang, Z. Melatonin derivatives combat with inflammation-related cancer by targeting the Main Culprit STAT3. Eur. J. Med. Chem. 2021, 211, 113027. [CrossRef]

19. Moradkhani, F.; Moloudizargari, M.; Fallah, M.; Asghari, N.; Heidari Khoei, H.; Asghari, M.H. Immunoregulatory role of melatonin in cancer. J. Cell Physiol. 2020, 235, 745-757. [CrossRef]

20. Moroishi, T.; Hansen, C.G.; Guan, K.L. The emerging roles of YAP and TAZ in cancer. Nat. Rev. Cancer 2015, 15, 73-79. [CrossRef]

21. Zanconato, F.; Cordenonsi, M.; Piccolo, S. YAP/TAZ at the Roots of Cancer. Cancer Cell 2016, 29, 783-803. [CrossRef]

22. Wang, Y.; Dong, Q.; Zhang, Q.; Li, Z.; Wang, E.; Qiu, X. Overexpression of yes-associated protein contributes to progression and poor prognosis of non-small-cell lung cancer. Cancer Sci. 2010, 101, 1279-1285. [CrossRef] 
23. Kuo, C.C.; Ling, H.H.; Chiang, M.C.; Chung, C.H.; Lee, W.Y.; Chu, C.Y.; Wu, Y.C.; Chen, C.H.; Lai, Y.W.; Tsai, I.L.; et al. Metastatic Colorectal Cancer Rewrites Metabolic Program Through a Glut3-YAP-dependent Signaling Circuit. Theranostics 2019, 9, $2526-2540$. [CrossRef]

24. Lee, K.Y.; Kuo, T.C.; Chou, C.M.; Hsu, W.J.; Lee, W.C.; Dai, J.Z.; Wu, S.M.; Lin, C.W. Upregulation of CD109 Promotes the Epithelial-to-Mesenchymal Transition and Stemness Properties of Lung Adenocarcinomas via Activation of the Hippo-YAP Signaling. Cells 2020, 10, 28. [CrossRef]

25. Lau, A.N.; Curtis, S.J.; Fillmore, C.M.; Rowbotham, S.P.; Mohseni, M.; Wagner, D.E.; Beede, A.M.; Montoro, D.T.; Sinkevicius, K.W.; Walton, Z.E.; et al. Tumor-propagating cells and Yap/Taz activity contribute to lung tumor progression and metastasis. EMBO J. 2014, 33, 468-481. [CrossRef]

26. Lee, W.Y.; Chen, P.C.; Wu, W.S.; Wu, H.C.; Lan, C.H.; Huang, Y.H.; Cheng, C.H.; Chen, K.C.; Lin, C.W. Panobinostat sensitizes KRAS-mutant non-small-cell lung cancer to gefitinib by targeting TAZ. Int. J. Cancer 2017, 141, 1921-1931. [CrossRef]

27. Pobbati, A.V.; Hong, W. A combat with the YAP/TAZ-TEAD oncoproteins for cancer therapy. Theranostics 2020, 10, 3622-3635. [CrossRef]

28. Kapoor, A.; Yao, W.; Ying, H.; Hua, S.; Liewen, A.; Wang, Q.; Zhong, Y.; Wu, C.J.; Sadanandam, A.; Hu, B.; et al. Yap1 activation enables bypass of oncogenic Kras addiction in pancreatic cancer. Cell 2014, 158, 185-197. [CrossRef]

29. Shao, D.D.; Xue, W.; Krall, E.B.; Bhutkar, A.; Piccioni, F.; Wang, X.; Schinzel, A.C.; Sood, S.; Rosenbluh, J.; Kim, J.W.; et al. KRAS and YAP1 converge to regulate EMT and tumor survival. Cell 2014, 158, 171-184. [CrossRef]

30. Zhang, W.; Nandakumar, N.; Shi, Y.; Manzano, M.; Smith, A.; Graham, G.; Gupta, S.; Vietsch, E.E.; Laughlin, S.Z.; Wadhwa, M.; et al. Downstream of mutant KRAS, the transcription regulator YAP is essential for neoplastic progression to pancreatic ductal adenocarcinoma. Sci. Signal 2014, 7, ra42. [CrossRef]

31. Chen, N.; Fang, W.; Lin, Z.; Peng, P.; Wang, J.; Zhan, J.; Hong, S.; Huang, J.; Liu, L.; Sheng, J.; et al. KRAS mutationinduced upregulation of PD-L1 mediates immune escape in human lung adenocarcinoma. Cancer Immunol. Immunother. 2017, 66, 1175-1187. [CrossRef]

32. Lan, B.; Ma, C.; Zhang, C.; Chai, S.; Wang, P.; Ding, L.; Wang, K. Association between PD-L1 expression and driver gene status in non-small-cell lung cancer: A meta-analysis. Oncotarget 2018, 9, 7684-7699. [CrossRef]

33. Scheel, A.H.; Ansen, S.; Schultheis, A.M.; Scheffler, M.; Fischer, R.N.; Michels, S.; Hellmich, M.; George, J.; Zander, T.; Brockmann, M.; et al. PD-L1 expression in non-small cell lung cancer: Correlations with genetic alterations. Oncoimmunology 2016, 5, e1131379. [CrossRef]

34. Skoulidis, F.; Byers, L.A.; Diao, L.; Papadimitrakopoulou, V.A.; Tong, P.; Izzo, J.; Behrens, C.; Kadara, H.; Parra, E.R.; Canales, J.R.; et al. Co-occurring genomic alterations define major subsets of KRAS-mutant lung adenocarcinoma with distinct biology, immune profiles, and therapeutic vulnerabilities. Cancer Discov. 2015, 5, 860-877. [CrossRef] [PubMed]

35. Li, J.; Chen, Y.; Shi, X.; Le, X.; Feng, F.; Chen, J.; Zhou, C.; Chen, Y.; Wen, S.; Zeng, H.; et al. A systematic and genome-wide correlation meta-analysis of PD-L1 expression and targetable NSCLC driver genes. J. Thorac. Dis. 2017, 9, 2560-2571. [CrossRef] [PubMed]

36. Cooper, W.A.; Tran, T.; Vilain, R.E.; Madore, J.; Selinger, C.I.; Kohonen-Corish, M.; Yip, P.; Yu, B.; O’Toole, S.A.; McCaughan, B.C.; et al. PD-L1 expression is a favorable prognostic factor in early stage non-small cell carcinoma. Lung Cancer 2015, 89, 181-188. [CrossRef] [PubMed]

37. Tang, Y.; Fang, W.; Zhang, Y.; Hong, S.; Kang, S.; Yan, Y.; Chen, N.; Zhan, J.; He, X.; Qin, T.; et al. The association between PD-L1 and EGFR status and the prognostic value of PD-L1 in advanced non-small cell lung cancer patients treated with EGFR-TKIs. Oncotarget 2015, 6, 14209-14219. [CrossRef] [PubMed]

38. Li, C.; Liu, J.; Xie, Z.; Zhu, F.; Cheng, B.; Liang, H.; Li, J.; Xiong, S.; Chen, Z.; Liu, Z.; et al. PD-L1 expression with respect to driver mutations in non-small cell lung cancer in an Asian population: A large study of 1370 cases in China. Ther. Adv. Med. Oncol. 2020, 12, 1758835920965840. [CrossRef]

39. Lo Sardo, F.; Muti, P.; Blandino, G.; Strano, S. Melatonin and Hippo Pathway: Is There Existing Cross-Talk? Int. J. Mol. Sci. 2017, 18, 1913. [CrossRef]

40. Zhao, X.; Sun, J.; Su, W.; Shan, H.; Zhang, B.; Wang, Y.; Shabanova, A.; Shan, H.; Liang, H. Melatonin Protects against Lung Fibrosis by Regulating the Hippo/YAP Pathway. Int. J. Mol. Sci. 2018, 19, 1118. [CrossRef]

41. Yang, C.E.; Lee, W.Y.; Cheng, H.W.; Chung, C.H.; Mi, F.L.; Lin, C.W. The antipsychotic chlorpromazine suppresses YAP signaling, stemness properties, and drug resistance in breast cancer cells. Chem. Biol. Interact 2019, 302, 28-35. [CrossRef]

42. van Rensburg, H.J.J.; Azad, T.; Ling, M.; Hao, Y.; Snetsinger, B.; Khanal, P.; Minassian, L.M.; Graham, C.H.; Rauh, M.J.; Yang, X. The Hippo Pathway Component TAZ Promotes Immune Evasion in Human Cancer through PD-L1. Cancer Res. 2018, 78, 1457-1470. [CrossRef]

43. Chung, C.H.; Lu, K.Y.; Lee, W.C.; Hsu, W.J.; Lee, W.F.; Dai, J.Z.; Shueng, P.W.; Lin, C.W.; Mi, F.L. Fucoidan-based, tumor-activated nanoplatform for overcoming hypoxia and enhancing photodynamic therapy and antitumor immunity. Biomaterials 2020, 257, 120227. [CrossRef]

44. Odeh, L.H.; Talib, W.H.; Basheti, I.A. Synergistic effect of thymoquinone and melatonin against breast cancer implanted in mice. J. Cancer Res. Ther. 2018, 14, S324-S330. [CrossRef]

45. Liu, H.; Xu, L.; Wei, J.E.; Xie, M.R.; Wang, S.E.; Zhou, R.X. Role of CD4+ CD25+ regulatory T cells in melatonin-mediated inhibition of murine gastric cancer cell growth in vivo and in vitro. Anat. Rec. 2011, 294, 781-788. [CrossRef] 
46. Perfilyeva, Y.V.; Ostapchuk, Y.O.; Abdolla, N.; Tleulieva, R.; Krasnoshtanov, V.C.; Belyaev, N.N. Exogenous Melatonin UpRegulates Expression of CD62L by Lymphocytes in Aged Mice under Inflammatory and Non-Inflammatory Conditions. Immunol. Investig. 2019, 48, 632-643. [CrossRef]

47. Cheng, L.; Liu, J.; Liu, Q.; Liu, Y.; Fan, L.; Wang, F.; Yu, H.; Li, Y.; Bu, L.; Li, X.; et al. Exosomes from Melatonin Treated Hepatocellularcarcinoma Cells Alter the Immunosupression Status through STAT3 Pathway in Macrophages. Int. J. Biol. Sci. 2017, 13, 723-734. [CrossRef]

48. Moreno, A.C.R.; Porchia, B.; Pagni, R.L.; Souza, P.D.C.; Pegoraro, R.; Rodrigues, K.B.; Barros, T.B.; Aps, L.; de Araujo, E.F.; Calich, V.L.G.; et al. The Combined Use of Melatonin and an Indoleamine 2,3-Dioxygenase-1 Inhibitor Enhances Vaccine-Induced Protective Cellular Immunity to HPV16-Associated Tumors. Front. Immunol. 2018, 9, 1914. [CrossRef]

49. Hadadi, E.; Acloque, H. Role of circadian rhythm disorders on EMT and tumour-immune interactions in endocrine-related cancers. Endocr. Relat. Cancer 2021, 28, R67-R80. [CrossRef]

50. Lee, K.Y.; Shueng, P.W.; Chou, C.M.; Lin, B.X.; Lin, M.H.; Kuo, D.Y.; Tsai, I.L.; Wu, S.M.; Lin, C.W. Elevation of CD109 promotes metastasis and drug resistance in lung cancer via activation of EGFR-AKT-mTOR signaling. Cancer Sci. 2020. [CrossRef] 